\title{
A Momentum-Based Indicator for Predicting the Peak Opening Load of Supersonic Parachutes
}

\author{
David W. Way \\ NASA Langley Research Center \\ Hampton, VA 23681-2199 \\ 757-864-8149 \\ david.w.way@nasa.gov
}

\begin{abstract}
In this paper, a new empirical indicator for predicting the peak opening loads of supersonic parachutes is presented. The proposed indicator is proportional to twice the free-stream dynamic pressure and the projected area of the parachute, which is equivalent to estimating the opening load as a percentage of the free-stream momentum flux through the projected area at the moment of peak inflation. The form of this expression is motivated by a classical control volume analysis of the aerodynamic forces acting on a parachute during inflation, under the simplifying assumptions of quasi-static and one-dimensional flow. For parachute geometries and flight conditions typical of Mars Entry, Descent, and Landing systems, the largest contribution to the total drag is shown to be a momentum flux term that is associated with the entrainment of atmosphere within the inflating parachute volume. Using this new method, empirical constants are calculated from existing flight reconstruction data and are shown to have a smaller standard deviation than similar constants determined using the customary indicator form, which is based on the steady-state subsonic drag and proportional to the parachute reference area. These empirical constants are also compared to an analytic estimate, derived from the control volume analysis, and shown to have excellent agreement across a wide range of Mach numbers and dynamic pressures for several parachute geometries. While opening loads estimated using both methods produce similar results at low supersonic Mach numbers typical of past inflations, the proposed method predicts notably larger loads at higher Mach numbers, those above Mach 2.0, due to the omission of any Mach Efficiency Factor. Several current Mars EDL projects have adopted this new indicator.
\end{abstract}

\section{Table of Contents}

1. INTRODUCTION...............................1

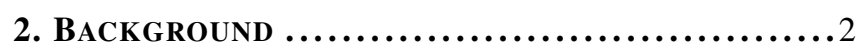

3. Effective Drag Coefficient, $C_{X} C_{D} \ldots \ldots \ldots . .2$

4. Momentum Constant, $k_{P} \ldots \ldots \ldots \ldots \ldots \ldots . . \ldots 4$

5. Results of a Control Volume Analysis......4

6. ANALYTIC ESTIMATE OF $k_{P} \ldots \ldots \ldots \ldots \ldots \ldots \ldots$

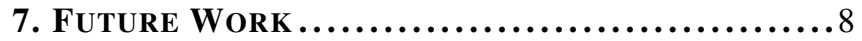

8. Conclusions ..................................8

ACKNOWLEDGMENTS ...........................9

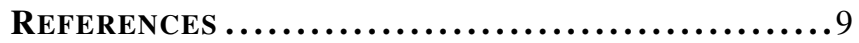

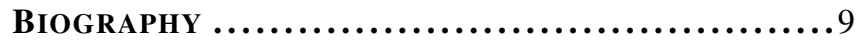

\section{INTRODUCTION}

The United States has successfully landed seven robotic systems on the surface of Mars. The earliest of which were

U.S. Government work not protected by U.S. copyright.

1 IEEE Aerospace Conference \#2817, Version 3, Updated December 13, 2017.

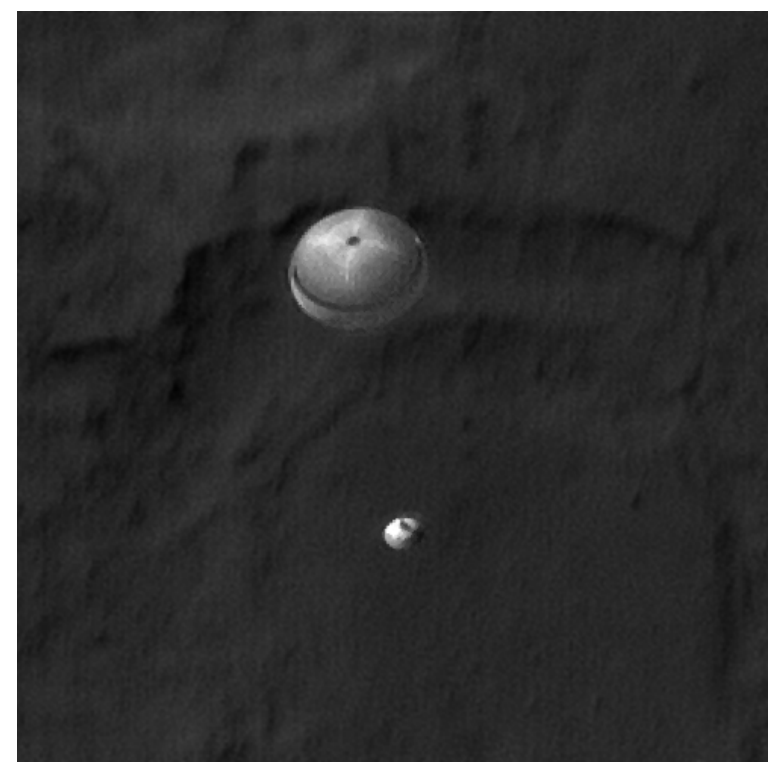

Figure 1. In 2012, the High-Resolution Imaging Science

Experiment (HiRISE) camera captured this image of Curiosity while descending on parachute at Mars. Image Credit: NASA/JPL-Caltech/Univ. of Arizona

the twin Viking landers (VL1 and VL2) in 1976. Twenty one years later, Mars Pathfinder (MPF) landed the Sojourner rover in 1997. This was followed in 2004 by the twin Mars Exploration Rovers (MER-A and MER-B) Spirit and Opportunity and in 2008 by the Mars Phoenix Lander (PHX). The latest landing, in 2012, was Mars Science Laboratory (MSL), carrying the Curiosity rover. A common denominator for all of these landings has been the Viking-derived Entry, Descent, and Landing (EDL) system architecture, which features a single mortar-deployed Disk-Gap-Band (DGB) supersonic parachute (See Figure 1). These parachutes perform the vital function of slowing the entry vehicle from supersonic terminal velocities to subsonic speeds, before too much altitude is lost. [1]

Not surprisingly, a key design parameter for these parachutes is the peak opening load and a key development activity is the structural qualification. Full-scale subsonic windtunnel tests, low-altitude drop tests, and/or high-altitude flight tests are needed to verify that the mechanical strength of the system is sufficient to survive the stresses applied at the design load with sufficient margin, as well as through subsequent repeated area oscillations. [2][3] In parallel, accurate predictions of the peak flight load are needed to ensure that the system will remain within the established operating conditions with a high degree of certainty. Indepen- 
dently, the canopy inflation behavior and drag performance are typically qualified by similarity to heritage high-altitude test flights, such as the Viking Balloon Launched Decelerator Test (BLDT) flights AV-2 and AV-4. [4]

However, the 2015 failure of a supersonic parachute during the Low Density Supersonic Decelerator (LDSD) second Supersonic Flight Dynamics Test (SFDT-2) [5] has since raised questions about the adequacy of the historically accepted methods by which these parachutes have been designed and tested in the past. In particular, the similarity and conservatism of stresses applied to the canopy during subsonic testing, as opposed to those applied in a supersonic flowfield have been questioned, prompting the creation of the Advanced Supersonic Parachute Inflation Research Experiment (ASPIRE) test program to supersonically qualify the Mars 2020 parachute on a high-altitude sounding rocket. In parallel, more accurate predictions of the peak loads were needed to support targeting of the ASPIRE test conditions and to bound the expected Mars 2020 flight loads.

In response, the Mars 2020 project has developed a new empirical opening load indicator for supersonic parachutes. The proposed indicator, Equation 1, is proportional to twice the free-stream dynamic pressure, $q_{\infty}$, and the projected area of the parachute, $S_{p r o j}$, which is equivalent to estimating the opening load, $F_{\text {peak }}$, as a percentage of the free-stream momentum flux through the projected area at the moment of peak inflation. Thus we refer to this indicator as "momentumbased" and the proportionality constant, $k_{P}$, as the "momentum constant".

$$
F_{\text {peak }}=2 k_{P} q_{\infty} S_{\text {proj }}
$$

\section{BACKGROUND}

The questions raised by the LDSD failure have prompted a renewed interest within the Mars EDL community to better understand the physics and predict the loads associated with supersonic parachute inflation. Yet, inflation dynamics of a parachute in a supersonic flow field is a very complex, transient, and chaotic system (See Figure 2 for an illustration of the complex geometries experienced during inflation), making analytical or even computational solutions extremely difficult. As Lingard explains, "it necessitates the solution of unsteady, separated, compressible flows about a flexible, porous body with non-uniform upstream flow conditions." [6] Even the most advanced Fluid-Structures Interaction (FSI) class of Computational Fluid Dynamics (CFD) codes are still considered unreliable in predicting or bounding the forces and stresses experienced in flight, though significant progress is being made. [7][8]

Various methods for modeling parachute inflation have been proposed in the literature. [9][10][11][12][13] These methods generally attempt to predict the evolution of the drag force throughout the inflation process (See Figure 3 for an example of typical load profile) and tend to fall into three categories: those that impose an inflation time, those that impose an inflation distance, or those that balance the forces in the radial direction. The wide variety of these methods again attests to the difficulty and complexity of the problem.

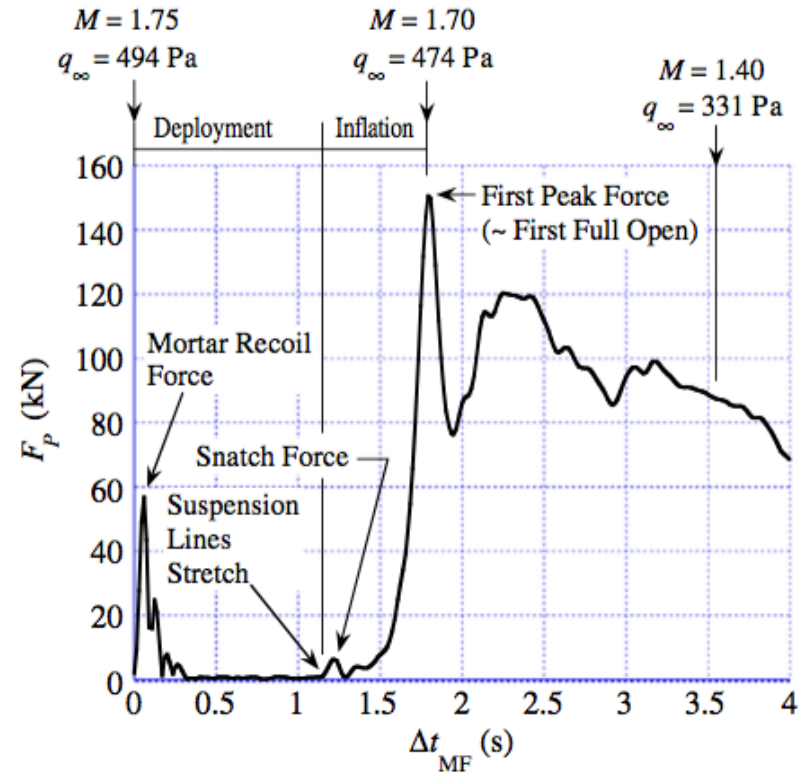

Figure 3. Reconstructed parachute force, $F_{P}$, as a function of time since mortar-fire, $\Delta t_{M F}$, from the Mars Science Laboratory (MSL) Entry, Descent, and Landing (EDL). Image Credit: Cruz et al. [14].

Many of these methods employ the concept of conservation of momentum and the authors emphasize the importance of the momentum transfer to the entrained atmosphere. Potvin even proposes a model based on the complete transfer of momentum to the entrained flow. [12]

In this paper, rather than attempting to precisely describe the geometry and internal pressures acting on the surface of the canopy, we instead take a more classic and macroscopic view of the problem. By applying the principles of conservation of mass and momentum to a control volume, we eliminate the need to describe exactly what is occurring within that control volume and must only account for the flux of mass and momentum entering and leaving the volume. The resulting expressions for the forces acting on the parachute, albeit simplified, provide some physical insight into the problem. These insights motivate the new empirical formulation for predicting peak opening loads, which we describe as momentum-based. We will compare the new indicator with the customary, standard approach and show that this new formulation agrees well with existing flight data.

\section{Effective Drag Coefficient, $C_{X} C_{D}$}

In this section, we provide a comparative baseline using the customary approach of estimating the opening load from an effective drag coefficient, which is the product of a constant over-inflation factor, $C_{X}$, and the steady-state drag coefficient, $C_{D}$. As shown in Equation 2, the steady-state drag coefficient at supersonic conditions is a function of Mach number only and is estimated by scaling the subsonic, incompressible, drag coefficient, $C_{D 0}$, by a Mach Efficiency Factor (MEF), $k_{M E F}$.[15] Figure 4 shows the Mach dependency of this factor, which peaks at approximately Mach $=1.5$ and decreases sharply for larger Mach numbers. For MSL the overinflation factor was estimated as $C_{X}=1.407$. Thus, the instantaneous peak opening load at $M=1.7\left(k_{M E F}=1.181\right)$ 


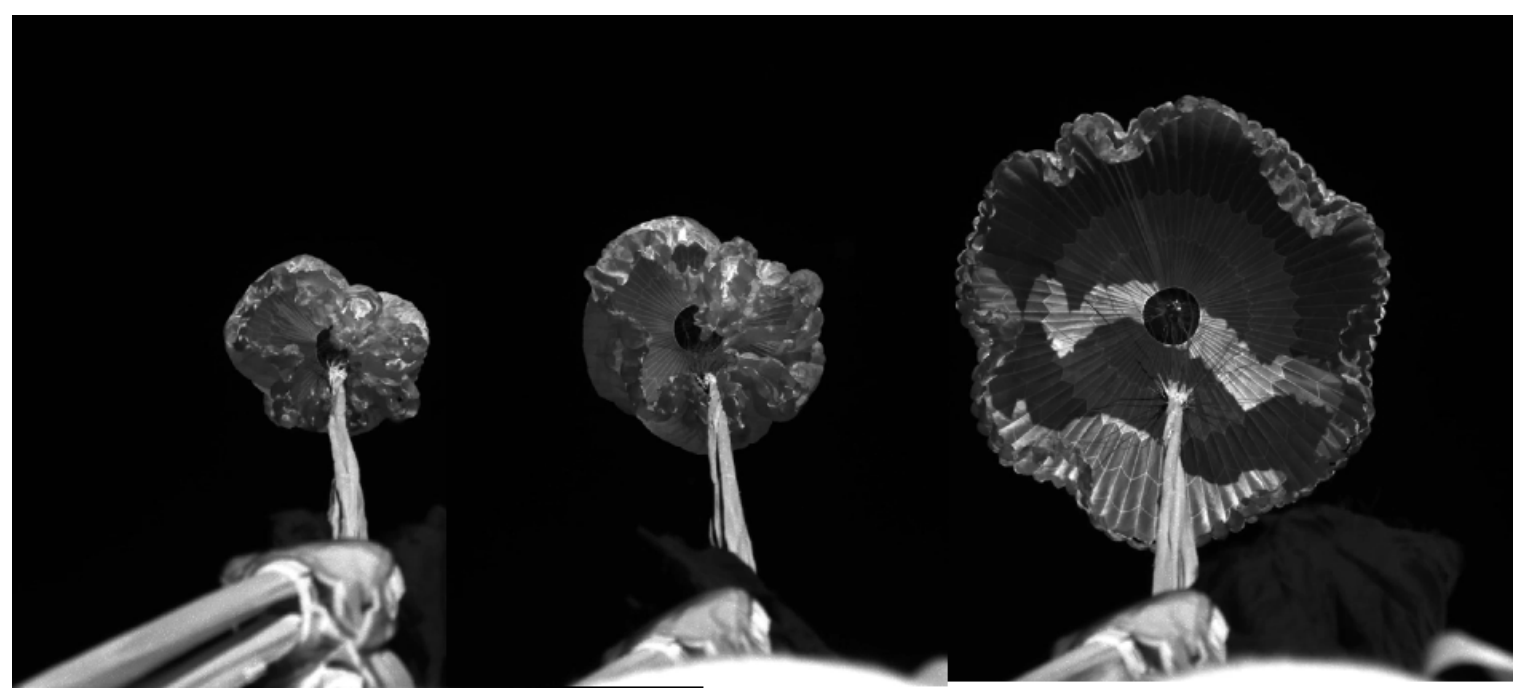

Figure 2. Three images extracted from high speed video of the parachute inflation sequence on the ASPIRE SR-01 test flight. The parachute is a $21.5 \mathrm{~m}$ MSL-heritage Disk-Gap-Band parachute. Image Credit: NASA/JPL-Caltech.

would be estimated by assuming an effective supersonic drag coefficient that was $66.2 \%$ higher than the steady-state, incompressible drag coefficient. where :

$$
F_{\text {peak }}=C_{X} C_{D} q_{\infty} S_{r e f}
$$

$C_{D}=k_{M E F} C_{D 0}$

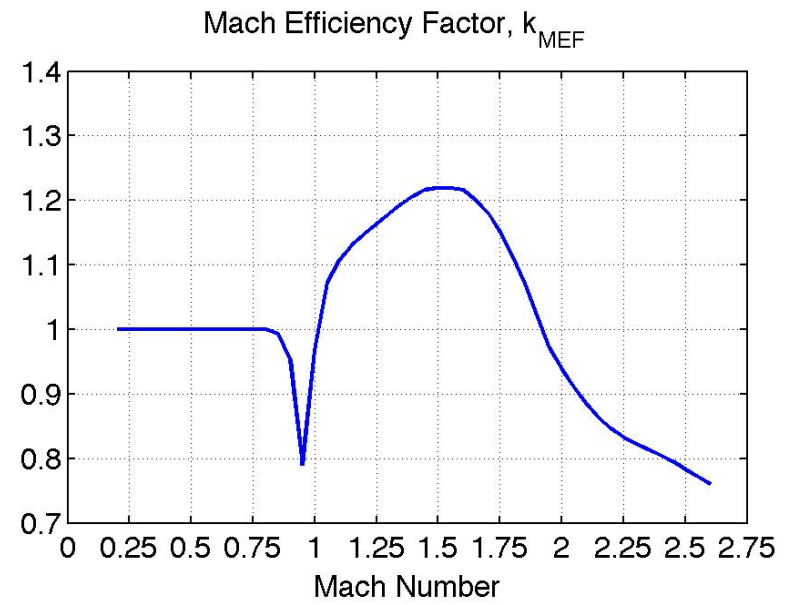

Figure 4. Mach Efficiency Factor (MEF) model for Mars Science Laboratory (MSL) Disk-Gap-Band (DGB) parachute.

In practice, the steady-state drag coefficient, $C_{D 0}$ and the MEF, $k_{M E F}$, are estimated from wind-tunnel data. [16] Then the over-inflation factor, $C_{X}$, is estimated from the empirically determined product of $C_{X} C_{D}$ as shown in Table 1 .

Table 1 provides reconstructed flight data from each of the seven Mars landings, along with a few high-altitude Earth
Table 1. Standard Empirical Constants Derived from Reconstructed Flight Data. $C_{X} C_{D}=\frac{F_{p e a k}}{q_{\infty} S_{r e f}}$

\begin{tabular}{|l|r|r|r|r|}
\hline Mission & $F_{\text {peak }}(k N)$ & $q_{\infty}(P a)$ & $S_{\text {ref }}\left(m^{2}\right)$ & $C_{X} C_{D}$ \\
\hline AV-2 & 40.0 & 221.4 & 204.85 & 0.882 \\
AV-4 & 72.0 & 408.4 & 204.85 & 0.861 \\
VL1 & 50.2 & 319.6 & 204.85 & 0.767 \\
VL2 & 49.8 & 329.0 & 204.85 & 0.739 \\
MPF & 35.5 & 588.0 & 126.68 & 0.477 \\
MER-A & $51.1^{3}$ & 718.8 & 156.15 & 0.455 \\
MER-B & $58.7^{3}$ & 762.2 & 156.15 & 0.493 \\
PHX & 44.9 & 466.0 & 109.36 & 0.881 \\
MSL & 153.8 & 474.0 & 363.05 & 0.894 \\
SFDT-2 & 386.9 & 557.0 & 736.81 & 0.943 \\
\hline Peak ope
\end{tabular}

Peak opening load, $F_{\text {peak }}$, is the maximum aerodynamic force. Dynamic pressure, $q_{\infty}$, is estimated for the time at peak load. Sample statistics: $\mu_{C_{X} C_{D}}=0.739 . \sigma_{C_{X} C_{D}}=0.192$.

flight tests. ${ }^{2}$ This table provides the reconstructed opening loads, $F_{\text {peak }}$, and dynamic pressure at full-inflation, $q_{\infty}$, along with the reference area, $S_{r e f}$, for each canopy. From these data we can estimate the product $C_{X} C_{D}$.

These data, plotted graphically in Figure 5, exhibit a good bit of variability. The sample standard deviation from Table 1 is $\sigma=0.192$., which is approximately $26 \%$ of the mean value, $\mu=0.739$. The primary reason for this variability is the various parachute geometries, all of which have different steady-state drag coefficients. AV-1, AV-2, VL1, VL2, PHX, and MSL are all Viking-geometry DGBs; MPF is a modified Viking DGB with a $2 \mathrm{x}$ band width; MER-A and MER-B are DGBs with a 1.6x Viking band width; and SFDT-2 is a ring-

${ }^{2}$ Significant uncertainty exists in many of the values contained in this table. Opening loads reported in the literature often don't specify the assumptions under which the value was reconstructed. Also, parachute deployment conditions are often reported only at mortar-fire, rather than at full-inflation.

${ }^{3}$ Opening loads for MER-A and MER-B are likely underestimated due to the low sampling frequency $(8 \mathrm{~Hz})$ of the telemetered flight data. [17] 


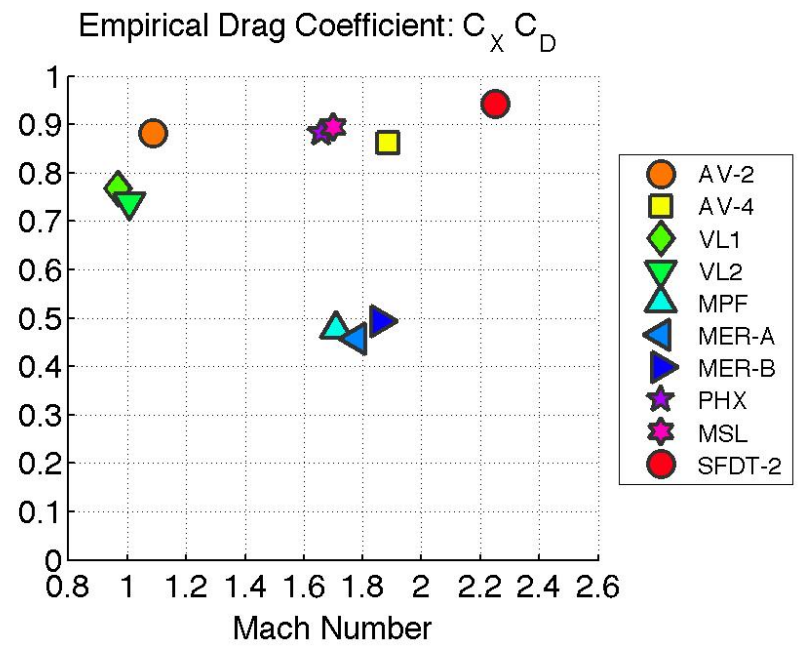

Figure 5. Standard empirical drag coefficients, $C_{X} C_{D}$, plotted versus Mach number. Data from Table 1.

Table 2. Proposed Empirical Constant Derived from Reconstructed Flight Data. $k_{P}=\frac{F_{p e a k}}{2 q_{\infty} S_{p r o j}}$

\begin{tabular}{|l|r|r|r|r|}
\hline Mission & $F_{\text {peak }}(k N)$ & $q_{\infty}(P a)$ & $S_{\text {proj }}\left(m^{2}\right)$ & $k_{P}$ \\
\hline AV-2 & 40.0 & 221.4 & 110.36 & 0.819 \\
AV-4 & 72.0 & 408.4 & 110.36 & 0.799 \\
VL1 & 50.2 & 319.6 & 110.36 & 0.712 \\
VL2 & 49.8 & 329.0 & 110.36 & 0.686 \\
MPF & 35.5 & 588.0 & 39.02 & 0.774 \\
MER-A & $51.1^{4}$ & 718.8 & 58.50 & 0.608 \\
MER-B & $58.7^{4}$ & 762.2 & 58.50 & 0.658 \\
PHX & 44.9 & 466.0 & 58.92 & 0.818 \\
MSL & 153.8 & 474.0 & 195.60 & 0.829 \\
SFDT-2 & 386.9 & 557.0 & 425.58 & 0.816 \\
\hline
\end{tabular}

Peak opening load, $F_{\text {peak }}$, is the maximum aerodynamic force. Dynamic pressure, $q_{\infty}$, is estimated for the time at peak load. Sample statistics: $\mu_{k_{P}}=0.752 . \sigma_{k_{P}}=0.080$.

sail design.

\section{Momentum Constant, $k_{P}$}

As we did for $C_{X} C_{D}$ in the previous section, we estimate our new empirical constant, $k_{P}$, from the same available flight data. These data are provided in Table 2, and shown graphically in Figure 6.

The only difference is that we now normalize the observed opening load by $2 S_{p r o j}$, rather than $S_{r e f}$. Since, for a hemispherical parachute, the reference projected area is approximately twice the projected area, we find that the numerical values of $k_{P}$ and $C_{X} C_{D}$ are similar. However, note that the sample standard deviation, $\sigma=0.080$, is significantly reduced. This result implies that peak opening load scales more closely with projected area, $S_{\text {proj }}$, than it does with reference area, $S_{r e f}$ - at least for this class of parachute.

\footnotetext{
${ }^{4}$ Opening loads for MER-A and MER-B are likely underestimated due to the
} low sampling frequency $(8 \mathrm{~Hz})$ of the telemetered flight data. [17]
Empirical Momentum Constant: $\mathrm{k}_{\mathrm{P}}$

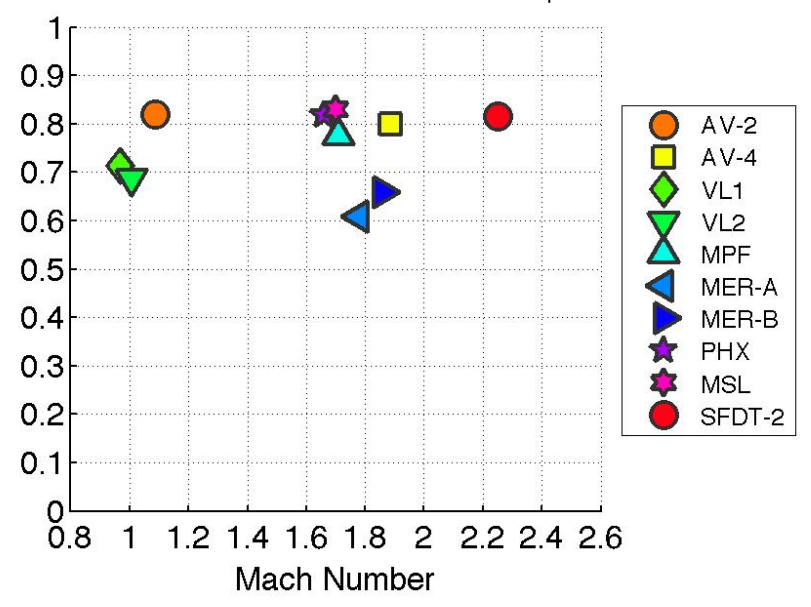

Figure 6. Proposed empirical momentum constants, $k_{P}$, plotted versus Mach number. Data from Table 2.

\section{Results of a Control Volume ANALYSIS}

The following section briefly presents the results a control volume analysis, which was performed for the purpose of deriving simplified expressions for the forces acting on a supersonic parachute during inflation. In subsequent sections, these expressions will then be used to motivate our chosen form for the opening load indicator and to provide a reasonable analytic estimate of the magnitude of the proportionality constant, $k_{P}$. In this analysis, the principles of conservation of mass and momentum where applied to an imaginary volume enclosing the parachute in order to find the net aerodynamic force acting on the parachute, $F_{d r a g}$.

This analysis was conducted, under the simplifying assumptions of quasi-static and quasi-one-dimensional flow, in the same manner in which the thrust of a jet engine is often derived, as in [18]. Equation 3, is the classic equation for the thrust of a ramjet engine. The first term, $\dot{m}_{\text {exit }} u_{\text {exit }}$, is called the "gross thrust" and is the flux of exhaust product momentum leaving the nozzle. The second term, $\dot{m}_{i n} u_{\infty}$, is called the "ram drag" and is the flux of free-stream momentum entering the inlet. The last term, $\left(p_{\text {exit }}-p_{\infty}\right) S_{\text {exit }}$, is called the "pressure correction", which accounts for the static pressure acting on the nozzle exit plane.

$$
\begin{aligned}
F_{\text {thrust }} & =\dot{m}_{\text {exit }} u_{\text {exit }}-\dot{m}_{i n} u_{\infty} \\
& +\left(p_{\text {exit }}-p_{\infty}\right) S_{\text {exit }}
\end{aligned}
$$

As Anderson points out [18], it is important to note that the physical mechanism by which the working fluid reacts with our system is by exerting pressure and shear stress on the internal and external surfaces. However the net result of this interaction is that momentum is transferred on a molecular level from the working fluid to the system as a result of the elastic collisions occurring between the fluid particles and the threads of the parachute fabric. We use this fact to compute the aerodynamic force from the change in momentum of the atmosphere as it travels through our control volume, since the momentum gained or lost by the fluid must be balanced by an 
equal and opposite change in the momentum of the system. We use this approach because it is more simple and more practical than attempting to solve for the precise distribution of pressure over the entire surface area of the parachute.

We start with presenting a free-body diagram of our system, Figure 7, which depicts the forces and accelerations acting on the parachute. From this we write Equation 4, which is Newton's 2nd Law, where $\vec{F}_{\text {tension }}$ is the external tension force applied by the parachute suspension system onto the canopy, $\vec{F}_{d r a g}$ is the net aerodynamic force acting on the parachute, and $\vec{F}_{\text {buoy }}$ is the buoyancy force due to the displacement of free-stream atmosphere by denser entrained atmosphere within the volume of the parachute. The vector sum of these forces is equal to the product of sensed acceleration and the mass of the system, which includes both the physical mass of the parachute, $m_{\text {chute }}$, along with the mass of the entrained atmosphere, $m_{\text {entr }}$.

$$
\vec{F}_{\text {tension }}+\vec{F}_{\text {buoy }}+\vec{F}_{\text {drag }}=\left(m_{\text {chute }}+m_{\text {entr }}\right) \vec{a}_{\text {sens }}
$$

The magnitude of the buoyancy force is equal to the weight of the displaced fluid, $F_{\text {buoy }}=\rho_{\infty} V_{\text {entr }} g$, and the axial component of this force is $\rho_{\infty} V_{\text {entr }} g \sin \bar{\gamma}$. This force acts against the weight of the entrained atmosphere, $\rho_{e a} V_{\text {entr }} g \sin \bar{\gamma}$, so that the net buoyancy is small. For supersonic inflations at Mars (or Mars-like conditions for high-altitude Earth tests), where the atmospheric density, $\rho_{\infty}$, is quite low, the magnitude of the buoyancy term is often neglected, but is included here for completeness. ${ }^{5}$ The effect of the buoyancy can also be neglected when the flight path angle is horizontal, $\bar{\gamma}=0$, as is the case in typical wind-tunnel testing. Thus, when neglecting both the buoyancy and entrained mass, and noting that the parachute is designed to be a deceleration device (i.e. $a_{\text {sens }}<0$ ), Equation 4 reduces to Equation 5 .

$$
\begin{gathered}
\quad F_{\text {tension }} \approx F_{\text {drag }}-m_{\text {chute }}\left|a_{\text {sens }}\right| \\
\text { when }: \\
F_{\text {buoy }} \approx 0 \\
m_{\text {entr }} \ll m_{\text {chute }} \\
a_{\text {sens }}<0
\end{gathered}
$$

The above equation is important for two reasons. First, note that the tension is equal to the drag (i.e. $F_{\text {tension }}=F_{d r a g}$ ) only when the acceleration is exactly zero, as is the case in a wind-tunnel experiment. In that case, the aerodynamic force $F_{d r a g}$ can be directly measured by recording the reaction forces in load measuring devices placed in the suspension system or on the sting. Otherwise, when the parachute is decelerating, the tension force that would be measured by load pins is less than the aerodynamic force (i.e. $F_{\text {tension }}<$ $\left.F_{d r a g}\right)$ by an amount equivalent to the inertial load on the parachute: $m_{\text {chute }}\left|a_{\text {sens }}\right|$. This effect can be significant, amounting to thousands of pounds of force. Secondly, the mere fact that these loads are different creates confusion

\footnotetext{
${ }^{5}$ Note that this buoyancy term may be large and non-negligible in the case of very large parachutes operating in thick atmospheres, such as for low-altitude Earth testing of Mars parachutes.
}

in the interpretation of published opening loads, since the literature often fails to specify which of these forces is being reported. ${ }^{6}$

Equation 6 is the continuity equation written for a control volume surrounding our parachute system, where $\dot{m}_{e n t r}$ is the time rate of change of entrained atmosphere within the control volume. This equation simply states that $\dot{m}_{e n t r}$ is equal to the net difference between inflow and outflow mass fluxes, $\dot{m}_{i n}$ and $\dot{m}_{\text {exit }}$. Note that during inflation, the mass of the system is not constant, (i.e. $\dot{m}_{\text {entr }}>0$ ), which differs significantly from the steady-state, post-inflation state.

$$
\dot{m}_{e n t r}=\dot{m}_{i n}-\dot{m}_{e x i t}
$$

Similarly, Equation 7 is the conservation of momentum applied to the control volume. This equation states that the aerodynamic force is equal to the sum of the net flux of momentum into the control volume, the time rate of change of momentum within the control volume, and the net pressure forces acting on the faces of the control volume.

$$
\begin{aligned}
F_{\text {drag }} & =\dot{m}_{\text {in }} u_{\infty}-\dot{m}_{\text {exit }} u_{\text {exit }} \\
& -\dot{m}_{\text {entr }} u_{\text {trans }} \\
& -\left(p_{\text {exit }}-p_{\infty}\right) S_{\text {exit }} \\
& -\left(p_{\text {wake }}-p_{\infty}\right)\left(S_{\text {in }}-S_{\text {exit }}\right)
\end{aligned}
$$

Comparing Equation 7 with Equation 3, we see many common terms, noting that each term in Equation 3 appears negated in Equation 7, since the drag and thrust are defined as positive in opposing directions. However, Equation 7 adds two additional terms that are not present in Equation 3 - one momentum term and one pressure term. The third term, $\dot{m}_{\text {entr }} u_{\text {trans }}$, is the time rate of change of momentum of the entrained atmosphere. This term would be zero for a ramjet since the mass of the working fluid within the engine is constant. The final term, $\left(p_{\text {wake }}-p_{\infty}\right)\left(S_{\text {in }}-S_{\text {exit }}\right)$, is an additional pressure correction term due to the static pressure in the parachute wake acting on the external surface of the parachute.

Finally, Figure 8 qualitatively compares the magnitude of each term in Equation 7 for a representative case of a $21.5 \mathrm{~m}$ DGB parachute opening at MSL flight conditions. ${ }^{7}$ The "inlet momentum flux" term, , $\dot{m}_{i n} u_{\infty}$, which is equivalent to the "ram drag" term in Equation 3, is far and away the dominant term. The magnitude of this term even exceeds the magnitude of the total drag, since most of the other contributions are negative. The only other positive term is the wake pressure correction, since the pressure in the wake is less than freestream pressure (i.e. $C_{p_{\text {wake }}}<0$ ).

Because the inlet momentum flux is by far the dominate term, and since its magnitude exceeds the total drag, it is reasonable to estimate the total drag as an empirical fraction of the inlet

${ }^{6}$ Flight reconstruction data reported in Table 1 and Table 2 of this paper assumes that the reported opening loads are aerodynamic forces (i.e. $F_{d r a g}$ ). In the event this assumption is not true, the actual opening load would be larger and may be contributing to some of the variability observed in the available data.

${ }^{7} \mathrm{Mach}=1.7$ and $q_{\infty}=474 P a$ in Mars atmosphere. 


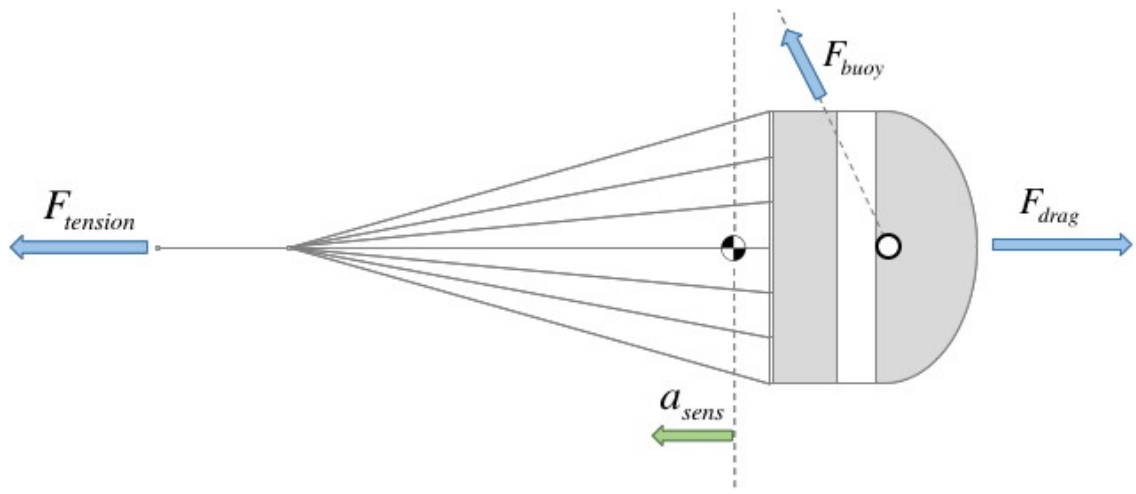

Figure 7. Free-body diagram depicting the positive directions of the sensed forces and accelerations acting on a parachute in flight. The buoyancy force is the weight of the free-stream atmosphere displaced by the denser entrained atmosphere within the parachute volume. Because the parachute is decelerating, the sensed acceleration is negative and acts in the opposite direction of the arrow shown here.

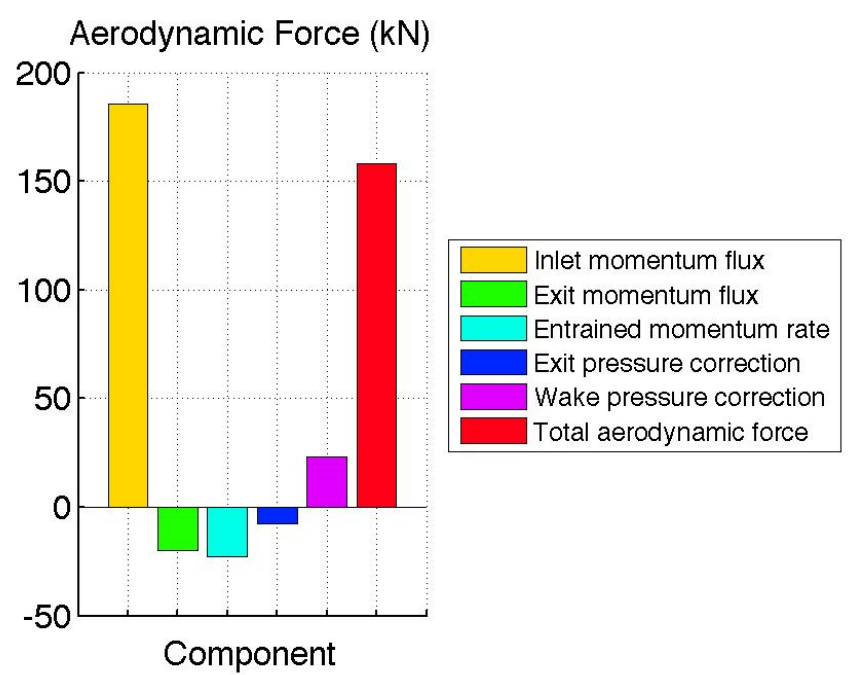

Figure 8. Relative contributions of each term in Equation 7 for a case representative of an MSL DGB at Mars. The $\dot{m}_{i n} u_{\infty}$ term, identified as "inlet momentum flux", exceeds the total drag, $F_{d r a g}$ (i.e. all other terms have a net negative contribution).

momentum flux, as shown in Equation 8. Substituting the identities $\dot{m}_{i n}=\rho_{\infty} u_{\infty} S_{i n}$ and $q_{\infty}=\frac{1}{2} \rho_{\infty} u_{\infty}^{2}$ we also find that $\dot{m}_{i n} u_{\infty}=2 q_{\infty} S_{i n}$.

$$
\begin{aligned}
F_{\text {drag }} & =k_{P}\left(\dot{m}_{i n} u_{\infty}\right) \\
& =k_{P}\left(2 q_{\infty} S_{i n}\right)
\end{aligned}
$$

Physically, this empirical momentum constant, $k_{P}$, is a nondimensional coefficient that describes the instantaneous fraction of the incoming momentum flux that is converted to aerodynamic drag. Taken at peak inflation, $k_{P}$ is the ratio of the peak opening load to the momentum flux through the projected area of the fully-inflated parachute, since $S_{i n}=$ $S_{\text {proj }}$ at full-inflation.

Another very important implication of Equation 8 is that we have removed the explicit Mach dependence, $k_{M E F}$. This implies that opening load is only a function of dynamic pressure, which is contrary to the assumed shape of the Mach efficiency factor, as shown in Figure 4. That figure suggests a decreasing opening load with increasing Mach number. As a consequence, it is reasonable to expect that $k_{P}$ might have an implicit Mach dependence. However, Figure 6, which plots the flight data of Table 2 against Mach number at fullinflation, shows very little variation over a wide range of Mach numbers. This issue will be addressed further in a subsequent section.

\section{Analytic Estimate of $k_{P}$}

In this section, we develop an analytical estimate for $k_{P}$ as a function of flight conditions and parachute geometry. Though this analysis may be extended to other regimes, we will make some assumptions that restrict our discussion to supersonic inflation. Given Equation 7 from the control volume analysis, we can substitute the parachute drag into Equation 8. This results in Equation 9.

$$
\begin{aligned}
k_{P} & =1-\left(\frac{\dot{m}_{\text {exit }}}{\dot{m}_{\text {in }}}\right)\left(\frac{u_{\text {exit }}}{u_{\infty}}\right) \\
& -\left(1-\frac{\dot{m}_{\text {exit }}}{\dot{m}_{\text {in }}}\right)\left(\frac{u_{\text {trans }}}{u_{\infty}}\right) \\
& -\frac{1}{2} C_{p_{\text {exit }}}\left(\frac{S_{\text {exit }}}{S_{\text {in }}}\right) \\
& -\frac{1}{2} C_{p_{\text {wake }}}\left(1-\frac{S_{\text {exit }}}{S_{\text {in }}}\right)
\end{aligned}
$$

We can now estimate each term in this expression by making a few assumptions. The general procedure is illustrated in Figure 9, while specific steps are enumerated below.

1. Assume a normal shock exists just inside the parachute canopy. This allows us to calculate post-shock and stagnation conditions inside the parachute from the normal shock relations (e.g. the post-shock Mach number, $M_{2}$, is found from Equation 10 where $M_{\infty}$ is the free-stream Mach number and $\gamma$ is the perfect-gas ratio of specific heats). 


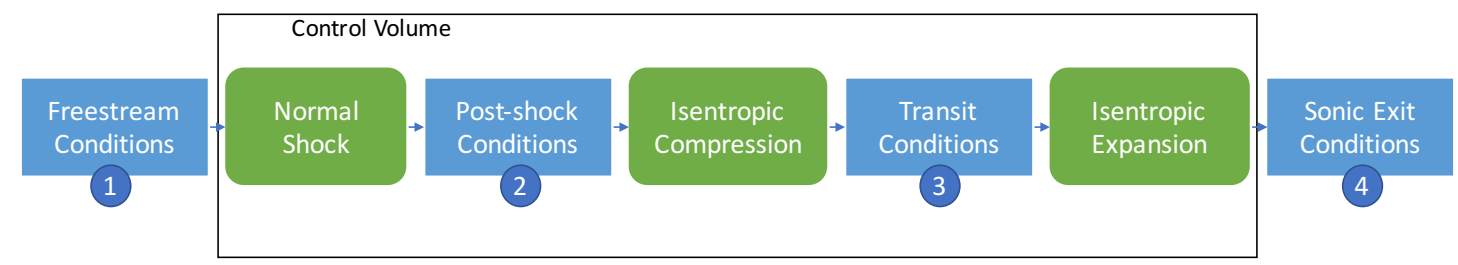

Figure 9. Assuming that a normal shock exists just inside the parachute canopy and that the exit flow is choked, normal shock relations and isentropic flow relations are used to estimate the flow conditions within the parachute control volume: (1) supersonic free-stream conditions, (2) subsonic post-shock conditions, (3) near-stagnation internal transiting flow conditions, and (4) sonic exit conditions.

$$
M_{2}=\sqrt{\frac{M_{\infty}^{2}(\gamma-1)+2}{2 \gamma M_{\infty}^{2}-(\gamma-1)}}
$$

Likewise, other post-shock properties are found by wellknown normal shock relationships (e.g. Equation 11 for pressure, Equation 12 for temperature, and Equation 13 for density).

$$
\begin{gathered}
\frac{p_{2}}{p_{\infty}}=\frac{2 \gamma M_{\infty}^{2}}{\gamma+1}-\frac{\gamma-1}{\gamma+1} \\
\frac{T_{2}}{T_{\infty}}=\frac{\left(1+\frac{\gamma-1}{2} M_{\infty}^{2}\right)\left(\frac{2 \gamma}{\gamma-1} M_{\infty}^{2}-1\right)}{M_{\infty}^{2}\left(\frac{2 \gamma}{\gamma-1}+\frac{\gamma-1}{2}\right)} \\
\frac{\rho_{2}}{\rho_{\infty}}=\frac{(\gamma+1) M_{\infty}^{2}}{(\gamma-1) M_{\infty}^{2}+2}
\end{gathered}
$$

The post-shock stagnation, or "total", conditions are found from the following relationships (e.g. Equation 14 for total pressure, Equation 15 for total temperature, and Equation 16 for total density).

$$
\begin{aligned}
\frac{p_{2_{0}}}{p_{2}} & =\left(1+\frac{\gamma-1}{2} M_{2}^{2}\right)^{\frac{\gamma}{\gamma-1}} \\
\frac{T_{2_{0}}}{T_{2}} & =\left(1+\frac{\gamma-1}{2} M_{2}^{2}\right) \\
\frac{\rho_{2_{0}}}{\rho_{2}} & =\left(1+\frac{\gamma-1}{2} M_{2}^{2}\right)^{\frac{1}{\gamma-1}}
\end{aligned}
$$

The inlet mass flow rate is given by Equation 17.

$$
\dot{m}_{i n}=\rho_{\infty} S_{i n} u_{\infty}
$$

2. Assume that the Mach number of the entrained atmosphere, as it transits through the parachute control volume, $M_{3}$, is a fixed fraction of $M_{2}$ (Equation 18). This allows us to calculate the properties of the entrained atmosphere from isentropic relations. Because the fraction $f_{\text {trans }}$ must be assumed, this value becomes an empirical parameter that may be adjusted to match observed data.

$$
\begin{array}{r}
M_{3}=f_{\text {trans }} M_{2} \\
0 \leq f_{\text {trans }} \leq 1
\end{array}
$$

Other post-shock properties are found from the post-shock stagnation conditions by well-known isentropic relations (e.g. Equation 19 for pressure, Equation 20 for temperature, and Equation 21 for density).

$$
\begin{aligned}
& \frac{p_{3}}{p_{2_{0}}}=\left(1+\frac{\gamma-1}{2} M_{3}^{2}\right)^{\frac{-\gamma}{\gamma-1}} \\
& \frac{T_{3}}{T_{2_{0}}}=\left(1+\frac{\gamma-1}{2} M_{3}^{2}\right)^{-1} \\
& \frac{\rho_{3}}{\rho_{2_{0}}}=\left(1+\frac{\gamma-1}{2} M_{3}^{2}\right)^{\frac{-1}{\gamma-1}}
\end{aligned}
$$

The transiting flow velocity, $u_{\text {trans }}$, can then be found from Equation 22.

$$
u_{\text {trans }}=M_{3} \sqrt{\gamma R T_{3}}
$$

3. Assume the exit flow is choked (i.e. $M_{\text {exit }}=1$ ) and that the ratio of exit to inlet areas is approximately equal to the porosity of the parachute, $\lambda$ (i.e. $S_{\text {exit }} \approx \lambda S_{\text {proj }}$ ). This allows us to calculate the properties of the exit flow from isentropic relations (e.g. Equation 23 for pressure, Equation 24 for temperature, and Equation 25 for density).

$$
\begin{aligned}
& \frac{p_{\text {exit }}}{p_{2_{0}}}=\left(1+\frac{\gamma-1}{2}\right)^{\frac{-\gamma}{\gamma-1}} \\
& \frac{T_{\text {exit }}}{T_{2_{0}}}=\left(1+\frac{\gamma-1}{2}\right)^{-1} \\
& \frac{\rho_{\text {exit }}}{\rho_{2_{0}}}=\left(1+\frac{\gamma-1}{2}\right)^{\frac{-1}{\gamma-1}}
\end{aligned}
$$

The exit flow velocity, $u_{e x i t}$, can then be found from Equation 26.

$$
u_{\text {exit }}=c_{\text {exit }}=\sqrt{\gamma R T_{\text {exit }}}
$$

The exit pressure coefficient is given by Equation 27.

$$
C_{p_{\text {exit }}}=\frac{\left(p_{\text {exit }}-p_{\infty}\right)}{q_{\infty}}
$$

The exit mass flow rate is given by Equation 28 .

$$
\dot{m}_{\text {exit }}=\rho_{\text {exit }} S_{\text {exit }} u_{\text {exit }}
$$




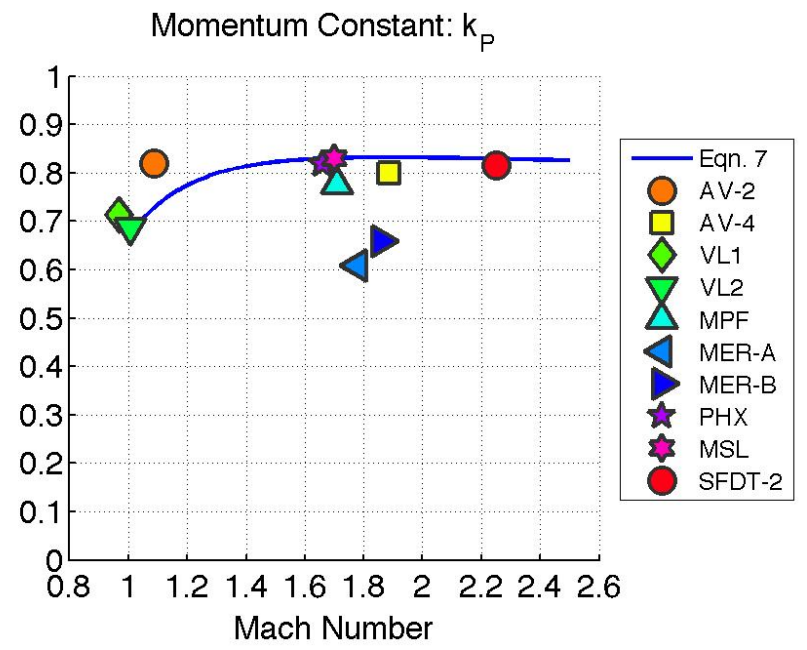

Figure 10. Comparison of analytic estimates from Equation 9 (assuming MSL inputs and $f_{\text {trans }}=0.4$ ) with Table 2 empirical data, showing excellent agreement between analytic and empirical results.

4. Finally, assume $C_{p_{\text {wake }}}$ is given empirically by the Viking back-pressure correction ${ }^{8}$ as developed by Mitcheltree (Equation 29). This allows us to estimate the pressure correction on the outer surface of the parachute canopy.

$$
\begin{aligned}
C_{p_{\text {wake }}}=A_{0} & +\frac{A_{1}}{M_{\infty}}+\frac{A_{2}}{M_{\infty}^{2}}+\frac{A_{3}}{M_{\infty}^{3}} \\
A_{0} & =0.008325 \\
A_{1} & =0.112933 \\
A_{2} & =-1.801004 \\
A_{3} & =1.288481
\end{aligned}
$$

Figure 10 plots the results of Equation 9 versus Mach number for an assumed transit Mach number fraction of $f_{\text {trans }}=$ $40 \%$. This estimate shows excellent agreement with the flight data, even capturing the slightly lower $k_{P}$ of the Viking landers as well as the $k_{P}$ for the SFDT-2 ringsail parachute at $M=2.26$.

Returning to the discussion of the Mach dependence of $k_{P}$, Figure 10 implies there may be some sensitivity at lower Mach numbers, near $M=1$, but shows a very flat trend at Mach numbers above $M=1.5$. Of course, this observation assumes that $f_{\text {trans }}$ is constant over all Mach numbers, as we assumed it to be, or at least doesn't vary significantly. Thus, it would be folly to place too much faith in this observation without additional data at higher Mach numbers.

\section{FUTURE WORK}

The proposed opening load indicator has been adopted by the Mars program projects currently under development, Mars 2020 and Insight, and is being used to help target desired

\footnotetext{
${ }^{8}$ As suggested by M. Schoenenberger in unpublished work, "Prediction of
} Peak Drag Coefficient During Inflation of a Supersonic Parachute," 2016. test conditions for the ASPIRE test flights. These upcoming flight opportunities will provide critical data for evaluating the effectiveness of a priori load estimates using the new indicator.

In particular the first ASPIRE flight (SR-01) will fly a buildto-print MSL parachute at MSL flight conditions, thus evaluating the similarity of $k_{P}$ between Earth flight tests and Mars flights. The second flight (SR-02) will fly a strengthened parachute at the same flight conditions, thus demonstrating any differences in load due to the differences in construction for geometrically similar parachutes. The third and fourth flights (SR-03 and SR-04) will target conditions at twice the MSL flight load, which will test the application of $k_{P}$ at higher dynamic pressures.

\section{Conclusions}

In this paper we propose a new empirical model for predicting peak opening loads in supersonic parachutes, $F_{\text {peak }}=$ $2 k_{P} q_{\infty} S_{p r o j}$. This model is informed by a derivation, from first principles, of the aerodynamic forces acting on a parachute during inflation. A classical control volume analysis was performed in which the conservation equations for mass and momentum were written, under the assumptions of quasi-static and one-dimensional flow.

Empirical constants calculated from existing flight reconstruction data were compared to those calculated in the customary way and were shown to have a much smaller standard deviation. These data were also compared to an analytic estimate with excellent agreement across a wide range of Mach numbers, dynamic pressures, and various parachute geometries and sizes.

The following particular observations are made:

1. Parachutes operate in very different flow regimes that may be characterized by whether or not the flow field is supersonic, $M>1$, and whether or not the mass of the entrained atmosphere is constant, $\dot{m}_{\text {entr }}>0$. This paper focused on only the region of supersonic inflation, and developed expressions for the opening load explicitly for these conditions and directly from first principles. This is in contrast to the customary approach, in which an estimate of the fully-inflated, steady-state, incompressible drag is used as the basis for the opening load prediction.

2. During the inflation of a supersonic parachute, the aerodynamic drag is dominated by the momentum exchanged with the entrained atmosphere. An analogy was made with the thrust produced by a ramjet engine and the two equations were shown to contain several identical terms. The largest term, larger even that than the total drag, was the term identified as the "ram drag", which is the momentum flux through the inlet area. This led to the approach of estimating the opening load as an empirical fraction of the incoming momentum flux, $2 q_{\infty} S_{p r o j}$.

3. Both the analytic estimate of $k_{P}$ and the empirical estimates, from flight reconstruction data, suggest that there may exist very little Mach dependence in the opening load, except possibly below $M=1.5$. This is in stark contrast with the customary approach, which predicts a significant reduction in opening load for increasing Mach numbers, based on the Mach efficiency factor, $k_{M E F}$. However, additional flight data at higher Mach numbers would be 
required to resolve this discrepancy in models.

\section{ACKNOWLEDGMENTS}

The author would like to thank Juan Cruz, Mark Schoenenberger, Soumyo Dutta, Eric Queen, Clara O'Farrell, and Ian Clark for their insight and guidance in reviewing previous revisions of this document.

\section{REFERENCES}

[1] R. D. Braun and R. M. Manning, "Mars Exploration Entry, Descent and Landing Challenges," Journal of Spacecraft and Rockets, Vol. 44, No. 2 (2007), pp. 310323.

[2] J. L. Raper, F. C. Michel, and R. R. Lundstrom, "The Viking Parachute Qualification Test Technique," AIAA 1973-456, 4th AIAA Aerodynamic Declerator Systems Technology Conference, Palm Springs, CA, May 1973.

[3] D. S. Adams and T. P. Rivellini, "Mars Science Laboratory's Parachute Qualification Approach," AIAA 20092913, 20th AIAA Aerodynamic Declerator Systems Technology Conference, Seattle, WA, May 2009.

[4] R. D. Moog and F. C. Michel, "Balloon Launched Viking Decelerator Test Program Summary Report," NASA-CR112288, TR-3720359, Mar. 1973.

[5] C. O'Farrell, E. J. Brandeau, C. Tanner, J. C. Gallon, S. Muppidi, and I. G. Clark, "Reconstructed Parachute System Performance During the Second LDSD Supersonic Flight Dynamics Test," AIAA 2016-3242, AIAA Atmospheric Flight Dynamics Conference, Washington, DC, June 2016.

[6] J. S. Lingard, M. G. Darley, and J. C. Underwood, "Simulation of Mars Supersonic Parachute Performance and Dynamics," AIAA 2007-2507, 19th AIAA Aerodynamic Declerator Systems Technology Conference, Williamsburg, VA, May 2007.

[7] J. R. Cruz and J. S. Lingard, "Aerodynamic Declerators for Planetary Exploration: Past, Present, and Future," AIAA 2006-6792, AIAA Guidance, Navigation, and Control Conference, Keystone, CO, Aug. 2006.

[8] A. Sengupta, A. Steltzner, A. Witkowski, G. Candler, and C. Pantano, "Findings from the Supersonic Qualificaiton Program of the Mars Science Laboratory Parachute System," AIAA 2009-2900, 20th AIAA Aerodynamic Declerator Systems Technology Conference, Seattle, WA, May 2009.

[9] B. W. Roberts and K. R. Reddy, "A Discussion of Parachute Inflation Theories," AIAA 1975-1351, 5th AIAA Aerodynamic Declerator Systems Technology Conference, Albuquerque, NM, Nov. 1975.

[10] D. Wolf, "A Simplified Dynamic Model of Parachute Inflation," AIAA 1973-450, 4th AIAA Aerodynamic Declerator Systems Technology Conference, Palm Springs, CA, May 1973.

[11] J. M. Macha, "A Simple, Approximate Model of Parachute Inflation," AIAA 1993-1206, Aerospace Design Conference, Irvine, CA, 1933.

[12] J. Potvin, "General Mass Capture Model for Swiftly Opening Parachutes,' Journal of Aircraft, Vol. 45, No. 5 (2008), pp. 1689-1700.
[13] J. Potvin and G. Peek, "Peak Load of a Drogue: Applying the Momentum-Impulse Theorem to Infinite Mass Inflation," AIAA 2015-2181, 23rd AIAA Aerodynamic Declerator Systems Technology Conference, Datona Beach, FL, Mar. 2015.

[14] J. R. Cruz, D. W. Way, J. D. Shidner, J. L. Davis, D. S. Adams, and D. M. Kipp, "Reconstruction of the Mars Science Laboratory Parachute Performance and Comparison to the Descent Simulation," Journal of Spacecraft and Rockets, Vol. 51, No. 4 (2014), pp. 1185-1196.

[15] J. R. Cruz, D. W. Way, J. D. Shidner, J. L. Davis, R. W. Powell, D. M. Kipp, D. S. Adams, A. Sengupta, M. Kandis, and A. Witkowski, "Parachute Models Used in the Mars Science Laboratory Entry, Descent, and Landing Simulation," AIAA 2013-1276, 26th AIAA Aerodynamic Declerator Systems Technology Conference, Datona Beach, FL, Mar. 2013.

[16] J. R. Cruz, R. E. Mineck, D. F. Keller, and M. V. Bobskill, "Wind Tunnel Testing of Various Disk-Gap-Band parachutes," AIAA 2003-2129, 17th AIAA Aerodynamic Declerator Systems Technology Conference, Monterey, CA, May 2003.

[17] A. Witkowski and M. Kandis, "Mars Exploration Rover Parachute System Performance," AIAA 2005-1605, 18th AIAA Aerodynamic Declerator Systems Technology Conference, Munich, Germany, May 2005.

[18] J. D. Anderson, "Introduction to Flight," 3rd Edition, McGraw-Hill, 1989.

\section{BIOGRAPHY}

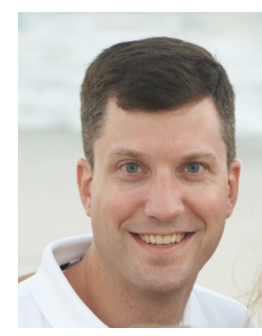

David Way received his Ph.D. in Aerospace Engineering from the Georgia Institute of Technology in 2001. Prior to that, he received a M.S. degree in Aerospace Engineering from Georgia Tech in 1997 and a B.S. degree in Aerospace Engineering from the $U$. S. Naval Academy in 1991. Assigned to the Atmospheric Flight and Entry Systems Branch of NASA's Langley Research Center, he is currently serving as the center's project lead for the Mars 2020 mission. In this role, he is responsible for the project's prime Entry, Descent, and Landing simulation. 